\title{
A three-dimensional picture of the delayed-detonation model of type la supernovae
}

\author{
E. Bravo ${ }^{1,2}$ and D. García-Senz ${ }^{1,2}$ \\ 1 Departament de Física i Enginyeria Nuclear, UPC, Jordi Girona 3, Mòdul B5, 08034 Barcelona, Spain \\ e-mail: eduardo.bravo@upc.edu \\ 2 Institut D'Estudis Espacials de Catalunya, Gran Capità 2-4, 08034 Barcelona, Spain \\ e-mail: domingo.garcia@upc.edu
}

Received 6 August 2007 / Accepted 7 November 2007

\begin{abstract}
Aims. Deflagration models poorly explain the observed diversity of SNIa. Current multidimensional simulations of SNIa predict a significant amount of, so far unobserved, carbon and oxygen moving at low velocities. It has been proposed that these drawbacks can be resolved if there is a sudden jump to a detonation (delayed detonation), but these kinds of models have been explored mainly in one dimension. Here we present new three-dimensional delayed detonation models in which the deflagraton-to-detonation transition (DDT) takes place in conditions like those favored by one-dimensional models.

Methods. We have used a smoothed-particle-hydrodynamics code adapted to follow all the dynamical phases of the explosion, with algorithms devised to handle subsonic as well as supersonic combustion fronts. The starting point was a centrally ignited $\mathrm{C}-\mathrm{O}$ white dwarf of $1.38 M_{\odot}$. When the average density on the flame surface reached $\sim 2-3 \times 10^{7} \mathrm{~g} \mathrm{~cm}^{-3}$ a detonation was launched.

Results. The detonation wave processed more than $0.3 M_{\odot}$ of carbon and oxygen, emptying the central regions of the ejecta of unburned fuel and raising its kinetic energy close to the fiducial $10^{51}$ erg expected from a healthy type Ia supernova. The final amount of ${ }^{56} \mathrm{Ni}$ synthesized also was in the correct range. However, the mass of carbon and oxygen ejected is still too high.

Conclusions. The three-dimensional delayed detonation models explored here show an improvement over pure deflagration models, but they still fail to coincide with basic observational constraints. However, there are many aspects of the model that are still poorly known (geometry of flame ignition, mechanism of DDT, properties of detonation waves traversing a mixture of fuel and ashes). Therefore, it will be worth pursuing its exploration to see if a good SNIa model based on the three-dimensional delayed detonation scenario can be obtained.
\end{abstract}

Key words. hydrodynamics - nuclear reactions, nucleosynthesis, abundances - shock waves - stars: evolution - white dwarfs supernovae: general

\section{Introduction}

The knowledge of the physical mechanism by which a white dwarf is disrupted by a thermonuclear explosion is relevant to many topics of modern astrophysics. A satisfactory model of the explosion becomes crucial to better understand type Ia supernovae, which in turn have profound implications in cosmology and in studies of the dynamics of the interstellar medium and the chemical evolution of galaxies. Nevertheless the details of the explosion mechanism are not well known. Nowadays the best models involve a white dwarf with a mass near the Chandrasekhar-mass limit in which a nuclear fuel ignites in one or many sparks around the center and thermonuclear combustion propagates rapidly through the rest of the star. In these models the starting point of hydrodynamical calculations is the thermal runaway caused by the screened ${ }^{12} \mathrm{C}+{ }^{12} \mathrm{C}$ nuclear reaction in degenerate conditions. The shape of the initially incinerated region probably does not keep the spherical symmetry that characterizes previous, hydrostatic, stages due to the stochastic nature of ignition and to the role played by hydrodynamic instabilities. As a consequence, any comprehensive numerical simulation of the explosion has to be performed in three dimensions. Many exploratory studies carried out in the past using onedimensional hydrodynamics have shown that the key point to achieve a successful explosion relies in the proper estimation of the velocity of the combustion front (Niemeyer \& Woosley 1997; Hillebrandt \& Niemeyer 2000). It has been shown (Timmes \& Woosley 1992) that the width of the flame is about $1 \mu \mathrm{m}$ at $\rho \simeq 10^{9} \mathrm{~g} \mathrm{~cm}^{-3}$, implying that at scales of a km the flame can be regarded as a thermal discontinuity separating ashes from fresh nuclear fuel. Although the flame velocity is large, close to $100 \mathrm{~km} \mathrm{~s}^{-1}$, it is quite subsonic: $\simeq 0.01 v_{\text {sound }}$, where $v_{\text {sound }}$ is the sound speed. These velocities are far below the nearly supersonic velocities which, according to many one-dimensional simulations, are needed to blow away the star. It is today widely accepted that the hydrodynamic instabilities inherent to the explosion are the drivers of combustion acceleration, thanks to the huge increase in the effective area of heat exchange between fuel and ashes.

Up to now the majority of three-dimensional studies of type Ia supernovae have focused on the pure deflagration paradigm (Garcia-Senz et al. 1998; Reinecke et al. 2002; Gamezo et al. 2003; García-Senz \& Bravo 2005; Schmidt \& Niemeyer 2006; Schmidt et al. 2006; Röpke et al. 2006a,b). In these models the flame front moves fast but always subsonically, leaving time for the white dwarf to expand before the deflagration reaches the outermost regions of the star. These models have been successful in explaining many observational facts using a 
minimum set of free parameters. Unfortunately, all the threedimensional versions of the pure deflagration paradigm present several weaknesses:

- The presence of a few tenths of unburnt, and unseen, carbon moving at low velocity is probably the worst flaw (Kozma et al. 2005).

- Although the amount of Fe-group elements synthesized in the explosion is within the expected range, the actual mass of ${ }^{56} \mathrm{Ni}$ ejected might turn out to be too low when neutronization due to electron captures is properly taken into account.

- The final kinetic energy never reaches the canonical value of 1 foe ( 1 foe $\equiv 10^{51} \mathrm{erg}$ ).

- The synthesis of intermediate-mass elements is scarce (see García-Senz et al. 2007).

- The ejecta are not chemically stratified, as demanded by observations of supernovae and remnants (Badenes et al. 2006; Gerardy et al. 2007).

- Large clumps of radioactive ${ }^{56} \mathrm{Ni}$ and ${ }^{56} \mathrm{Co}$ are present at the photosphere at the time of maximum brightness.

There have been several attempts to bypass the weaknesses of deflagrations models, preserving at the same time many of their advantages, by postulating a sudden transition from a subsonic deflagration to a supersonic detonation (Ivanova et al. 1974), i.e. the multidimensional version of the delayed detonation model: Khokhlov (1991) in 1D; Arnett \& Livne (1994); Livne (1999); Golombek \& Niemeyer (2005) in 2D; García-Senz \& Bravo (2003); Gamezo et al. (2004, 2005); Röpke \& Niemeyer (2007) in 3D. In particular, the recent work of Gamezo et al. (2005) gave a satisfactory thermonuclear explosion solving many of the problems of pure deflagration models. The density at which the jump to the detonation takes place had to be estimated because the physics behind the deflagration-to-detonation (DDT) transition is not well understood. In Gamezo et al. (2005) the adopted transition density was rather high, $\rho_{\mathrm{t}} \geq 2.5 \times 10^{8} \mathrm{~g} \mathrm{~cm}^{-3}$ and the detonation was induced near the center of the white dwarf. Röpke \& Niemeyer (2007) explored the range of explosions that would result if the DDT took place in the outer shells, at $r \sim 1.7-1.8 \times 10^{8} \mathrm{~cm}$, after a huge expansion drived by subsonic combustion initiated in a few hot bubbles. They used a physically motivated criterion to switch on a DDT, namely that the combustion entered the distributed regime, in which mixing of burnt and fresh fuel by turbulence fluctuations at the scale of the flame width might trigger a detonation. Although this criterion is as fragile as any other currently used in multidimensional simulations of DDT, it led to consistent results with respect to the final kinetic energy and to the mass of Fe-group and intermediate-mass elements synthesized. It also allowed almost complete depletion of carbon in the center.

Given the scarcity of three-dimensional simulations of the delayed detonation scenario and the complexity of the problem, it is interesting to perform additional calculations to explore further the range of initial conditions of the DDT. In this work we present new three-dimensional simulations of delayed detonations in white dwarfs in which the DDT takes place in conditions close to those usually assumed in one-dimensional calculations. In the present models the DDT was triggered when the mean density at the flame surface was $\rho \sim 2-3 \times 10^{7} \mathrm{~g} \mathrm{~cm}^{-3}$. We have also explored the sensitivity of the results to the initial geometrical configuration of the flame by computing a delayed detonation model in which the deflagrative phase started at the center of the white dwarf, and another model in which it started in 30 hot bubbles, as in the deflagration model B30U in García-Senz \& Bravo (2005).
The plan of the paper is as follows. In Sect. 2 we briefly describe the hydrocode and the physics included in the threedimensional simulations. Next, we discuss the hydrodynamical evolution during both the deflagration and the detonation stages, as well as the final nucleosynthesis. Some discussion of our results and a comparison to previous works as well as the conclusions are provided in Sect. 4.

\section{Methods}

The simulations were carried out using a smoothed-particle hydrodynamics (SPH) code suited to handle both subsonic deflagration waves (Garcia-Senz et al. 1998) and supersonic detonations (García-Senz et al. 1999). The calculations were carried out using 250000 particles, which translated into a maximum spatial resolution of $22 \mathrm{~km}^{1}$ (about $1 \%$ of the white dwarf radius) during the deflagration phase and, later on, of $15 \mathrm{~km}(\sim 0.2 \%$ of the stellar radius) during the detonation phase. The equation of state, the same as described in García-Senz \& Bravo (2005), includes all the terms relevant for the explosive phases of type Ia supernovae. The properties of matter in nuclear statistical equilibrium (NSE) were interpolated from accurate tables giving the nuclear binding energy, electron capture and neutrino generation rates as a function of the temperature, density and electron mole number. This represented a negligible overhead of CPU time and allowed us to obtain realistic estimates of the nucleosynthesis during the SNIa explosion. As usual in this kind of simulation, the detailed nucleosynthesis was calculated by postprocessing the output of the hydrodynamical model.

During the deflagration phase the flame was propagated by a diffusion-reaction scheme devised to match a prescribed flame velocity law. Although the SPH algorithms are flexible enough to incorporate a subgrid-scale model able to capture the effects of turbulent flows into the effective flame velocity (García-Senz et al. 2008, in preparation), for the present calculations we choose to stay as close to possible to the usual assumptions of succesful one-dimensional delayed detonation models. Thus, we implemented a constant flame velocity in the range $v_{\text {flame }}=$ $100-200 \mathrm{~km} \mathrm{~s}^{-1}$, independent of density, during the deflagration phase. As described in García-Senz \& Bravo (2005), this prescription has proven adequate to describe the gross features of subsonic burning propagation. Note that the true laminar velocity in the center of the white dwarf is close to $v_{\text {flame }} \simeq 100 \mathrm{~km} \mathrm{~s}^{-1}$. On the other hand the time-averaged effective deflagration velocity which stems from that scheme is similar to the one assumed in successful one-dimensional delayed detonation models during the deflagration phase, $\simeq 0.03 v_{\text {sound }}-0.05 v_{\text {sound }}$ (Domínguez \& Höflich 2000).

During the detonation phase the above described algorithm for flame propagation was switched off and the combustion was followed by solving the nuclear kinetic equations together with the hydrodynamic and energy equations. The nuclear part consisted of a small nuclear network of 9 nuclei from helium to nickel (Timmes et al. 2000) with prescriptions aimed to handle both quasi-statistical equilibrium and nuclear statistical equilibrium. Whenever the temperature became higher than $5.5 \times 10^{9} \mathrm{~K}$ the material was assumed to be incinerated to NSE if the density was higher than $10^{6} \mathrm{~g} \mathrm{~cm}^{-3}$. Once the NSE regime was attained the abundances were not computed by solving the nuclear kinetic equations, instead the chemical composition was obtained from

\footnotetext{
1 Here we give the smoothing length of the SPH code as a proxy for the spatial resolution. Note that the smoothing length is both spatially and temporally variable.
} 
Table 1. Main features of the models.

\begin{tabular}{|c|c|c|c|c|c|c|c|c|}
\hline Model & Ignition & $\begin{array}{c}E^{a} \\
\left(10^{51} \mathrm{erg}\right)\end{array}$ & $\begin{array}{c}M\left({ }^{56} \mathrm{Ni}\right) \\
\left(M_{\odot}\right)\end{array}$ & $\begin{array}{l}t_{\text {last }} \\
(\mathrm{s})\end{array}$ & $\begin{array}{c}I \\
\text { (Time steps) }\end{array}$ & $\begin{array}{c}\Delta E^{b} \\
\left(10^{51} \mathrm{erg}\right)\end{array}$ & $\begin{array}{c}\Delta p^{c} \\
\left(10^{9} M_{\odot} \mathrm{cm} \mathrm{s}^{-1}\right) \\
\end{array}$ & $\begin{array}{c}\Delta L^{d} \\
\left(10^{17} M_{\odot} \mathrm{cm}^{2} \mathrm{~s}^{-1}\right)\end{array}$ \\
\hline DDT3DA & Central volume & 0.75 & 0.54 & 7.9 & 7400 & 0.01 & $4 \times 10^{-8}$ & $2 \times 10^{-8}$ \\
\hline DDT3DB & 30 bubbles & 0.81 & 0.63 & 463. & 21200 & -0.008 & $1 \times 10^{-8}$ & $10^{-8}$ \\
\hline
\end{tabular}

${ }^{a}$ Final energy (gravitational+internal+kinetic) of the ejected mass at the last computed time, $t_{\text {last }}$.

${ }^{b}$ Accumulated error in the total energy at the last computed model, after $I$ time steps.

${ }^{c}$ Total momentum at the last computed model, normalized taking a characteristic velocity of the ejecta of $10^{9} \mathrm{~cm} \mathrm{~s}^{-1}$.

${ }^{d}$ Total angular momentum at the last computed model, normalized taking a characteristic velocity of the ejecta of $10^{9} \mathrm{~cm} \mathrm{~s}^{-1}$, and a characteristic radius of $10^{8} \mathrm{~cm}$.

the NSE tables. In our calculations, incinerated particles were assumed to leave NSE when either their temperature or their density were lower than $2 \times 10^{9} \mathrm{~K}$ or $10^{6} \mathrm{~g} \mathrm{~cm}^{-3}$, respectively. During this phase the detonation front was captured, that is the particles within the detonation front were identified with the aid of an algorithm that looked for simultaneous strong gradients of pressure and temperature together with sonic flows. Particles located at a detonation front were assigned ellipsoidal kernels to perform the SPH interpolations in such a way that the smallest axis of its ellipsoid was aligned with the normal to the detonation front. In regions where there were no shocks the kernel reduced to the standard spherically symmetric kernel. The ability of this device to represent detonation waves was demonstrated in Fig. 1 of García-Senz et al. (1999), where more details of the numerical method are available. Typically, these techniques lead to an improvement of a factor $\sim 2-3$ in spatial resolution.

The initial model consisted of an isothermal $\mathrm{C}-\mathrm{O}$ white dwarf of $1.38 M_{\odot}$ in hydrostatic equilibrium, with a central density $\rho_{\mathrm{c}}=1.8 \times 10^{9} \mathrm{~g} \mathrm{~cm}^{-3}$. We ran two simulations of the delayed detonation scenario, whose main features are given in Table 1. In model DDT3DA the first stages of the explosion were followed using a one-dimensional Lagrangian code until the mass burnt was $0.09 M_{\odot}$ and the central density had declined to $\rho_{\mathrm{c}}=1.4 \times 10^{9} \mathrm{~g} \mathrm{~cm}^{-3}$. At that point the structure was mapped to a $3 \mathrm{D}$ distribution of 250000 particles, and the radial velocity field at the flame location was perturbed by a sinusoid of wavenumber $l=20$ and amplitude $200 \mathrm{~km} \mathrm{~s}^{-1}$, as much as the velocity of matter behind the flame at that time. The ensuing evolution was followed with the three-dimensional SPH code, with a baseline velocity of $v_{\text {flame }}=100 \mathrm{~km} \mathrm{~s}^{-1}$. The initial geometry of the flame in model DDT3DB was different, consisting in a set of 30 sparks scattered around the center of the white dwarf. The three-dimensional calculation of this model started exactly as in model B30U of García-Senz \& Bravo (2005), to which we refer the reader for more details.

The switch from the subsonic combustion front to the supersonic regime also was handled in a different way in both models. In DDT3DA the detonation was induced simultaneously in many points on the flame surface, when its average density declined to $\sim 2 \times 10^{7} \mathrm{~g} \mathrm{~cm}^{-3}$. By contrast, in model DDT3DB the detonation was artificially triggered in a single region of the flame, although at a density similar to that in DDT3DA.

The last three columns of Table 1 give details of the performance of the code with respect to the conservation of total energy, momentum and angular momentum. The conservation of the two last is excellent, whereas that of energy is acceptable as the error stays around $1 \%$ of the final kinetic energy. Also supporting the ability of the code to handle the subsonic deflagration phase is the spherical symmetry retained by the pressure field during the huge expansion of the white dwarf in this phase, as shown in Fig. 1. Such a smooth pressure distribution has to

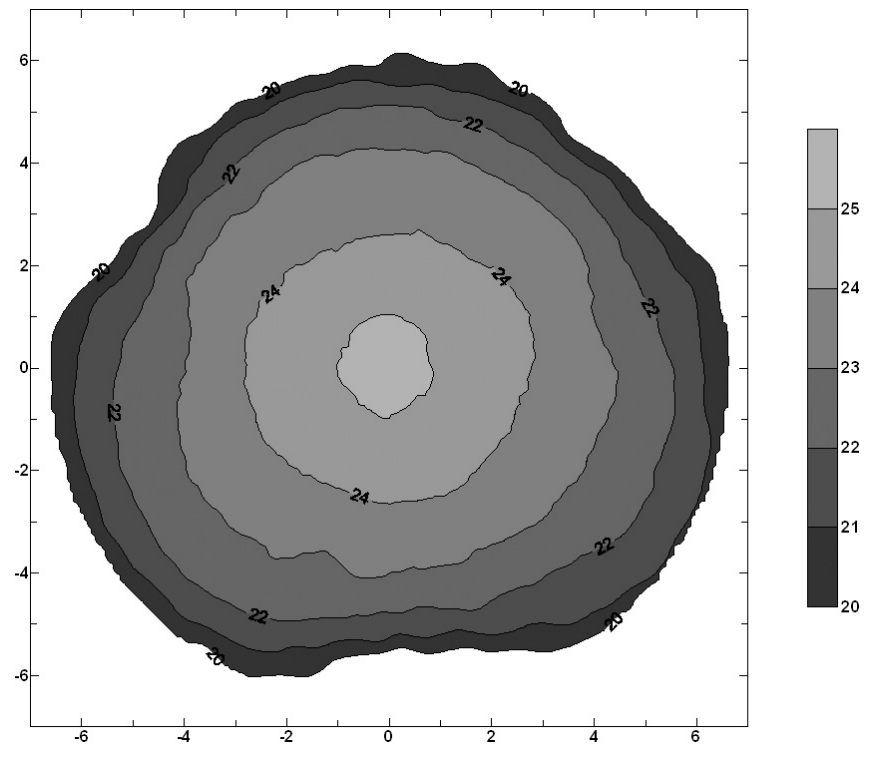

Fig. 1. Contour map of pressure in a cut across the star at the end of the deflagration phase of model DDT3DA $(t=1.55 \mathrm{~s})$. The labels of the isobars give the logarithm of pressure in $\mathrm{erg} \mathrm{cm}^{-3}$. The axis labels are in units of $1000 \mathrm{~km}$. The preservation of spherical symmetry is a consequence of the subsonic nature of the deflagration front.

be compared with the convoluted temperature field depicted in the last snapshot of Fig. 2. The limitations of our methodology to carry out simulations of type Ia supernovae were widely discussed in García-Senz \& Bravo (2005).

\section{Results of the three-dimensional simulations}

\subsection{Model DDT3DA: the deflagration phase}

This phase begins when the first sparks ignite in the central regions of the white dwarf and ends when the first detonation wave appears. Both modes of burning propagation can coexist for a while during the detonation phase although the efficiency of the deflagration is far below that of the supersonic detonation. As mentioned before, in our computations the deflagration algorithm was switched off once a DDT was artificially induced. Röpke \& Niemeyer (2007) have discussed the impact of subsonic burning after a DDT. They found that it is relevant only when the mass burnt during the deflagration phase is quite low as is the case, for instance, if the runaway starts in a few hot bubbles.

The geometry of the initial sparks which light the core of the white dwarf is not known. However, in the threedimensional realm it is to be expected that the ignition starts asynchronously in a number of small regions scattered around 

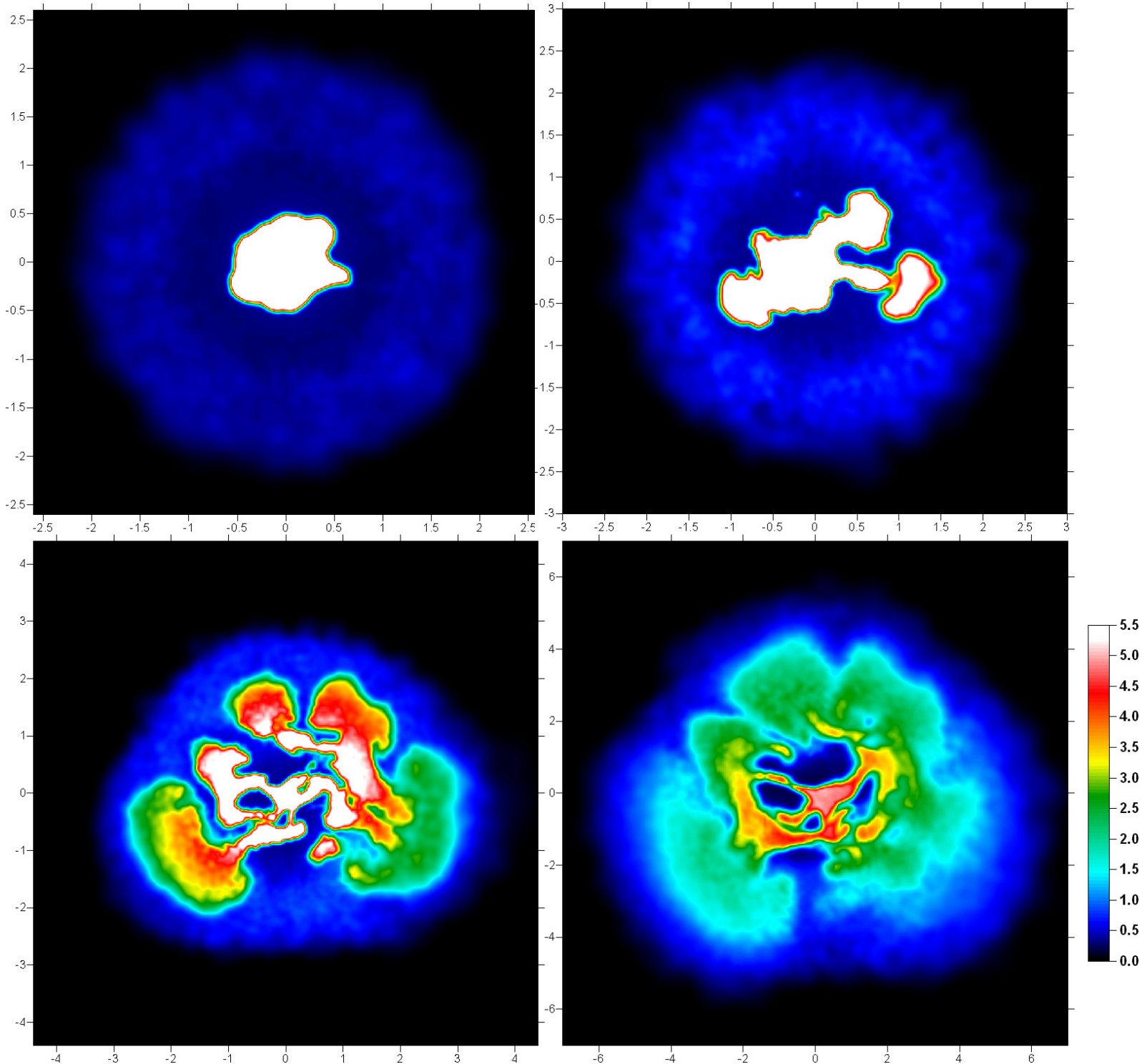

Fig. 2. Snapshots of the temperature map in a cut across model DDT3DA at times $t=0.56,0.86,1.20$, and $1.55 \mathrm{~s}$, all of them belonging to the deflagration phase. The lengthscale is given in units of $1000 \mathrm{~km} \mathrm{~s}^{-1}$, while the temperature scale is given in the side colorbar in units of $10^{9} \mathrm{~K}$.

the core (Garcia-Senz \& Woosley 1995; Wunsch \& Woosley 2004; Kuhlen et al. 2006). For a large enough number of igniting points located close to the center, bubbles of incinerated matter would merge before they have had the opportunity to detach from the burning pack. Were this the case, the hydrodynamical evolution would not be very different from that due to the massive ignition of the central volume of the core. This is the scenario we examine in model DDT3DA, in which the explosion was triggered by incinerating a small amount of mass in the central part of the core of an isothermal Chandrasekhar-mass white dwarf.

The evolution of the star during the deflagration phase can be seen in Figs. 2 and 3. In Fig. 2 there are several snapshots of the evolution from the beginning of the SPH calculation, which we define as $t=0 \mathrm{~s}$, when the flame surface was completely spherical, up to $t=1.55 \mathrm{~s}$, when the flame was strongly distorted by the Rayleigh-Taylor instability and the average density at the flame was $\left\langle\rho_{\text {flame }}\right\rangle \simeq 2 \times 10^{7} \mathrm{~g} \mathrm{~cm}^{-3}$. A complementary view is given in Fig. 3 where we plot the angle-averaged radial profiles of density, temperature, nuclear energy generation rate, and incinerated mass fraction during the deflagration phase. During the first half a second the flame front remained nearly spherical in spite of the sample of perturbations of the velocity field that were seeded at $t=0 \mathrm{~s}$. Afterwards, the fingers characteristic of the Rayleigh-Taylor instability developed very quickly (second and third snapshots in Fig. 2), which resulted in hot material rising and cool unburnt carbon and oxygen intruding towards the center (fourth panel of Fig. 3). As the flame surface increased due to the hydrodynamic instabilities, the rate of released nuclear energy also did. The effective burning velocity kept pace with the evolution of the surface, as can be seen in Fig. 4: it remained around $0.02 c_{\mathrm{s}}$ until $t=0.6 \mathrm{~s}$ and rose monotonically up to $0.14 c_{\mathrm{s}}$ at $t=1.4 \mathrm{~s}$. This large increase in the effective burning rate when $t \geq 0.6 \mathrm{~s}$ is as well apparent in the third panel of Fig. 3 which depicts the nuclear energy generation rate. At $t=0.86 \mathrm{~s}$ (second snapshot in Fig. 2) the burning front clearly displays the mushroom-like shape characteristic of the non-linear Rayleigh-Taylor regime, encompassing a large fraction of the white dwarf mass. As a consequence the rate of nuclear energy generation went up steeply until a maximum value of $1.3 \times 10^{51} \mathrm{erg} \mathrm{s}^{-1}$ was achieved at $t=1.1 \mathrm{~s}$. During the last second of deflagration propagation the average temperature 
Table 2. Main features of the models at the time of DDT.

\begin{tabular}{|c|c|c|c|c|c|c|c|c|}
\hline Model & 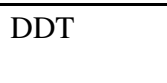 & $\begin{array}{l}t^{\mathrm{DDT}} \\
(\mathrm{s})\end{array}$ & $\begin{array}{c}\rho_{\mathrm{c}}^{\text {DDT }} \\
\left(10^{7} \mathrm{~g} \mathrm{~cm}^{-3}\right)\end{array}$ & $\begin{array}{c}\rho_{\mathrm{fl}}^{\text {DDT }} \\
\left(10^{7} \mathrm{~g} \mathrm{~cm}^{-3}\right)\end{array}$ & $\begin{array}{c}E_{\mathrm{tot}} \\
\left(10^{51} \mathrm{erg}\right)\end{array}$ & $\begin{array}{l}M_{\mathrm{NSE}}^{\mathrm{DDT}} \\
\left(M_{\odot}\right)\end{array}$ & $\begin{array}{c}M\left({ }^{56} \mathrm{Ni}\right)^{\mathrm{DDT}} \\
\left(M_{\odot}\right)\end{array}$ & $\begin{array}{c}M(\mathrm{C}-\mathrm{O})^{\mathrm{DDT}} \\
\left(M_{\odot}\right)\end{array}$ \\
\hline DDT3DA & Multipoint & 1.55 & 5.4 & 2.0 & 0.40 & 0.50 & 0.27 & 0.65 \\
\hline DDT3DB & Single point & 0.81 & 22.2 & 3.7 & 0.39 & 0.58 & 0.43 & 0.64 \\
\hline
\end{tabular}
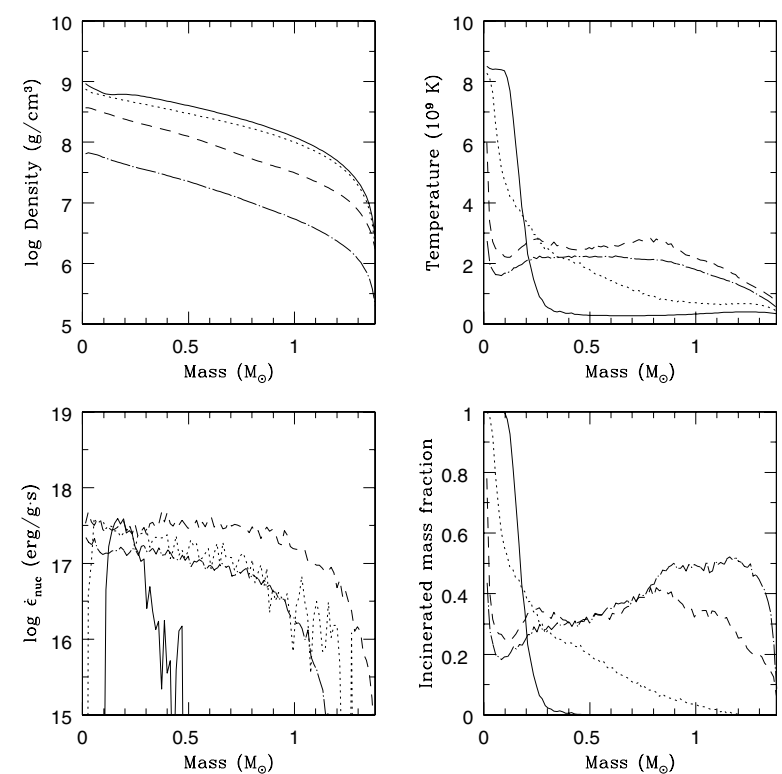

Fig. 3. Angle-averaged profiles of density, temperature, nuclear energy generation, and incinerated mass fraction during the deflagration phase. The lines represent the profiles at the same times as Fig. 2: $t=0.56 \mathrm{~s}$ (solid), $0.86 \mathrm{~s}$ (dotted), $1.20 \mathrm{~s}$ (short dashed), and $1.55 \mathrm{~s}$ (dot-long dashed).

and nuclear energy generation rate were uniform throughout the white dwarf.

When the elapsed time was $\sim 1.55 \mathrm{~s}$ the average density of the flame became low enough to allow intermediate-mass elements to be synthesized. At this time the central density had declined to $\rho_{\mathrm{c}} \simeq 5.4 \times 10^{7} \mathrm{~g} \mathrm{~cm}^{-3}$ and the expansion was fast enough that the nuclear reactions began freezing at the outermost regions of the deflagration front. The main features of the model at the end of this phase are summarized in Table 2. The amount of nickel synthesized, $0.27 M_{\odot}$, was too low to give a suitable explosion with the maximum luminosity of a typical SNIa. In addition, there remained too much carbon and oxygen.

The scaling properties of the flame surface during the deflagration phase were monitored, using the method described in Garcia-Senz et al. (1998), and the results are presented in Fig. 5 (filled dots). This kind of calculation, which gives the so-called correlation dimension, provides a purely geometric description of the flame structure determined by the spatial distribution of particles belonging to the flame. An independent way of obtaining the fractal dimension is to estimate the total flame surface within the minimum lengthscale resolved by the code, $h$, and the integral scale, using the ratio of the mass consumption rate, $\dot{M}$, and the baseline flame velocity (solid line in Fig. 5):

$D=2+\frac{\log \left(\dot{M} / 4 \pi \bar{r}^{2} \rho v_{\text {flame }}\right)}{\log (\bar{r} / h)}$

where the integral scale has been approximated by the average radius of the flame, $\bar{r}$. As it can be seen in Fig. 5 there is excellent agreement between both methods of computation

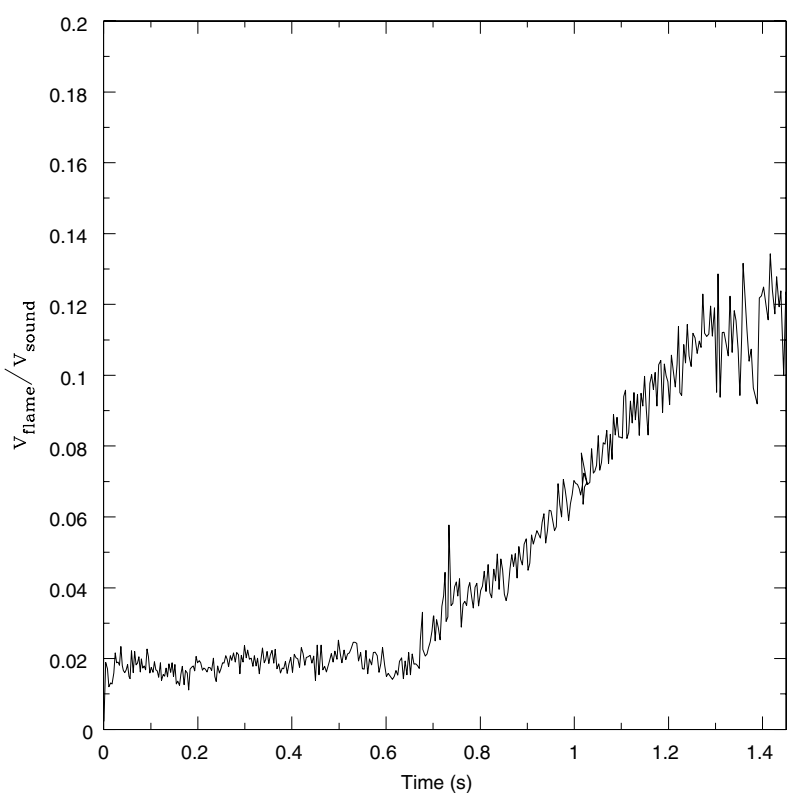

Fig. 4. Effective velocity of the deflagration front in units of the local sound speed as a function of time.

of the fractal dimension, implying that the above equation can be safely used in analytical and one-dimensional calculations to explore the physics of thermonuclear supernovae. After an initial period where the fractal dimension remained close to 2.0 it rapidly began to increase after $t=0.6 \mathrm{~s}$, at which time the average density of the flame had dropped below $5 \times 10^{8} \mathrm{~g} \mathrm{~cm}^{-3}$. Approximately $1.1 \mathrm{~s}$ after the beginning of the three-dimensional calculation the fractal dimension had reached a value $D \simeq 2.6$, similar to that associated with the RT instability in the non linear regime, $D_{\mathrm{RT}}=2.5$. That high value of the fractal dimension indicates that the Rayleigh-Taylor instability rather than turbulence ${ }^{2}$ was the dominant mechanism wrinkling the flame at those scalelengths directly resolved by the hydrocode. This was expected as our resolution is insufficient to resolve turbulence, i.e. the minimum smoothing length attained in the SPH calculation during deflagration was $20 \mathrm{~km}$ while the integral length of the turbulent cascade is $\sim 10 \mathrm{~km}$ (Lisewski et al. 2000). Afterwards, the fractal dimension saturated at $D \simeq 2.6-2.7$.

\subsection{Model DDT3DA: the detonation phase}

As has been discussed in Sect. 2, explosions driven purely by a deflagration usually run into trouble when compared to the observational properties of normal SNIa events for various reasons. One of them is the difficulty of reaching explosions with energies of roughly $10^{51} \mathrm{erg}$. Another serious objection is that current three-dimensional calculations always predict a strong

2 In the discussion we are assuming Kolmogorov turbulence (Zingale et al. 2005; Schmidt et al. 2006), characterized by a fractal dimension $D_{\mathrm{K}}=2.33$, although the turbulence might also be of the BolgianoObukov type, characterized by $D_{\mathrm{BO}}=2.6$ (Niemeyer \& Kerstein 2000). 


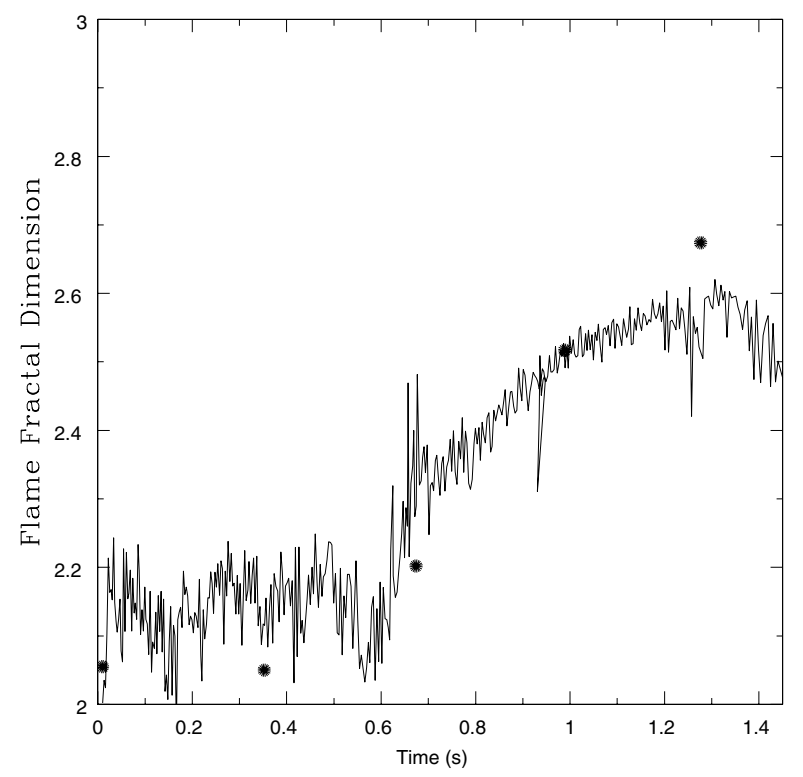

Fig. 5. Evolution of the fractal dimension of the flame. Dots show the correlation dimension, while the solid line represents an alternative estimate of the fractal dimension derived from the effective rate of mass burning.

mixing of elements in the ejecta. A possible way to circumvent these drawbacks is to assume that at some time the subsonic deflagration turns into a supersonic detonation at one or several points of the flame surface. Although several mechanisms have been suggested to account for such a transition, a definitive answer to this question is still missing. Therefore, the temporal and spatial coordinates at which the DDT takes place should be regarded as free parameters of the model (Livne 1999).

Many one-dimensional simulations carried out in the nineties suggested that the agreement with observations is fairly good when the transition takes place at densities around 2-4 $\times$ $10^{7} \mathrm{~g} \mathrm{~cm}^{-3}$. This is about the average density of the flame when its fractal dimension stabilizes around 2.6, and approaches the density at which combustion is thought to leave the flamelet regime and enter into the distributed regime. Let us assume that the flame propagates with the laminar velocity at the Gibson scale and that the flame surface behaves as a fractal of dimension 2.33 (a value determined by the turbulent energy spectrum) between the Gibson scale and the minimum scale resolved by the code, $h$. Then, the effective flame velocity can be obtained as a function of the fractal dimension, $D$, that characterizes the flame between $h$ and the integral scale. The result of this calculation is that the effective flame velocity exceeds the maximum velocity of a stable Chapman-Jouget deflagration (Khokhlov 1988) if $D>2.5$ at $\rho \lesssim 2 \times 10^{7} \mathrm{~g} \mathrm{~cm}^{-3}$. Thus, even though the laminar flame velocity always remains much lower than the sound velocity, the effective flame velocity can be high enough to compress the material ahead, switching on a detonation (Woosley \& Weaver 1994).

As a practical criterion to select the particles prone to detonate we flagged those placed at regions where the local fractal dimension was higher than $D=2.5$ when the average density of the flame was $\left\langle\rho_{\text {flame }}\right\rangle \simeq 2 \times 10^{7} \mathrm{~g} \mathrm{~cm}^{-3}$. As these high- $D$ regions trace the geometrical complexity of the flame it is not unreasonable to assume that they can harbor conditions suitable for detonation initiation. In our calculation the above criterion was satisfied for the first time at $t=1.55 \mathrm{~s}$, marking the end of the deflagration phase. A two-dimensional image of the flame

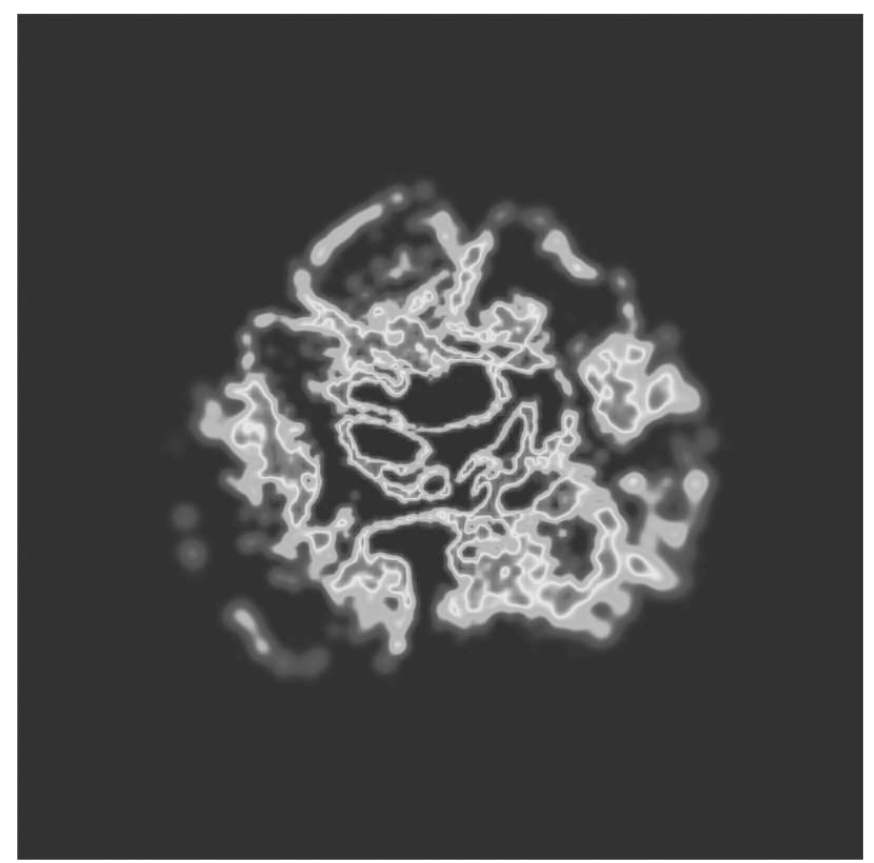

Fig. 6. Flame structure in a cut of the white dwarf at the end of the deflagration phase. The image shows the location of hot fuel whose temperature lies in the range $1-3 \times 10^{9} \mathrm{~K}$.

structure at this time is shown in Fig. 6, where its geometric complexity can be seen. The regions displaying the highest $D$ spanned the whole radial extent of the white dwarf, except for a narrow central volume and the outermost $0.2 M_{\odot}$. Once the high- $D$ particles were selected, a detonation was artificially induced through their instantaneous incineration. Note that we do not mean that reaching a given fractal dimension of the flame is a necessary or sufficient condition for transition to detonation, but for our purposes it provided a convenient way to select the time and location of the DDT.

As we did with the deflagration phase, we describe here the evolution during the detonation phase by means of two figures, Figs. 7 and 8, the first one showing several snapshots of the temperature field through a cut of the white dwarf, and the second one giving the evolution of the angle-averaged radial profiles of the fuel mass fraction. After a transient induction period, detonations settle in a steady state consisting of a shock plus a tiny reaction zone, followed by a much larger relaxation zone. Our code is only able to effectively resolve the last one. Thus, we cannot ascertain the result of the interaction of the detonation front with obstacles, i.e. inert volumes. However, matter incinerated to the NSE should not be described as inert, because at the densities we are considering it is able to react to the passage of a shock, that compress and heats matter, and readjust its composition accordingly (Maier \& Niemeyer 2006). Hence, when the precursor shock of a detonation transits through NSE matter, it rises its temperature favouring lighter, less bound, species, which in turn depletes energy from the shock front. In our calculations, the detonation did not survive the crossing of volumes composed of nuclear ashes. The complex geometry of the flame at the time of DDT favored the formation of isolated pockets of fuel that could not be caught by the detonation front, so that they remained unburned (Fig. 9).

Because the detonation was initiated in a more or less uniform way throughout the white dwarf, there followed a period of rapid and generalized combustion leading to a temperature 

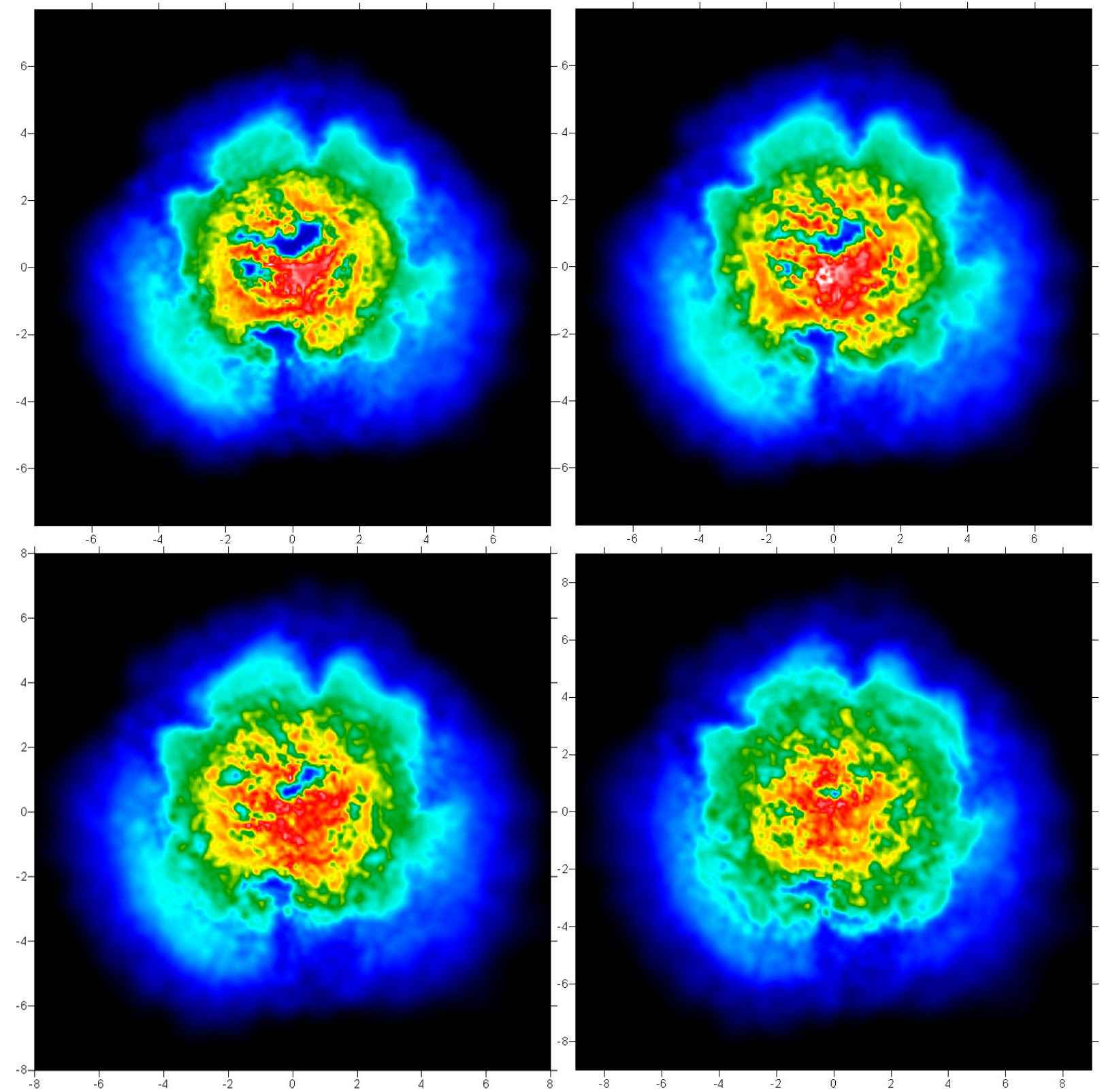

Fig. 7. Snapshots of the temperature map in a cut across model DDT3DA at times $t=1.58,1.61,1.66$, and $1.75 \mathrm{~s}$. The deflagration-to-detonation transition was induced just at $1.55 \mathrm{~s}$. The scales of length and temperature are the same as in Fig. 2.

distribution much more homogeneus than that of the preceding deflagration phase (Fig. 7). The inwards detonation wave synthesized an additional amount of ${ }^{56} \mathrm{Ni}$, whereas the outgoing detonation, travelling through lower density layers, synthesized intermediate-mass elements (Fig. 9). In this way, the DDT allowed the partial compensation of the shortcomings of the previous deflagration stage, giving an improved explosion model.

The main features of the explosion at the last computed time are given in Table 1, while the nucleosynthesis obtained by postprocessing the thermal history obtained in the hydrodynamical simulation is presented in Table 3 . The final kinetic energy is still on the low side but no longer very low whereas the amount of ${ }^{56} \mathrm{Ni}\left(0.54 M_{\odot}\right)$ is enough to power the light curve. Nevertheless, there still remains $0.39 M_{\odot}$ of unburnt carbon and oxygen, which should be compared with the $0.65 M_{\odot}$ left at the end of the deflagration phase. The reason why there still remains this large mass of carbon and oxygen is due to both the shielding effect of the ashes produced during the deflagration phase and the degradation of the code resolution when the detonation propagated through low-density regions. However, even if a much better resolution were achieved we would not expect major changes in the total energy of the ejecta or in the mass of ${ }^{56} \mathrm{Ni}$ synthesized.

The total amount of ${ }^{56} \mathrm{Fe}$ (after radioactive decay) and intermediate-mass elements ejected in the explosion are $0.66 M_{\odot}$ and $0.11 M_{\odot}$ respectively, which is of the same order as the abundances deduced from the spectra of normal type Ia supernovae and compatible with the demands of current models of galactic chemical evolution. Another positive feature of the model is that the ashes are not concentrated in large clumps as is usually obtained in pure deflagration models. In addition, there remains little unburnt carbon and oxygen close to the center. Unfortunately, model DDT3DA does not completely remove all nucleosynthetic deficiencies affecting current three-dimensional models of SNIa. There are too many Fe-group elements close to the surface of the white dwarf, especially too much 


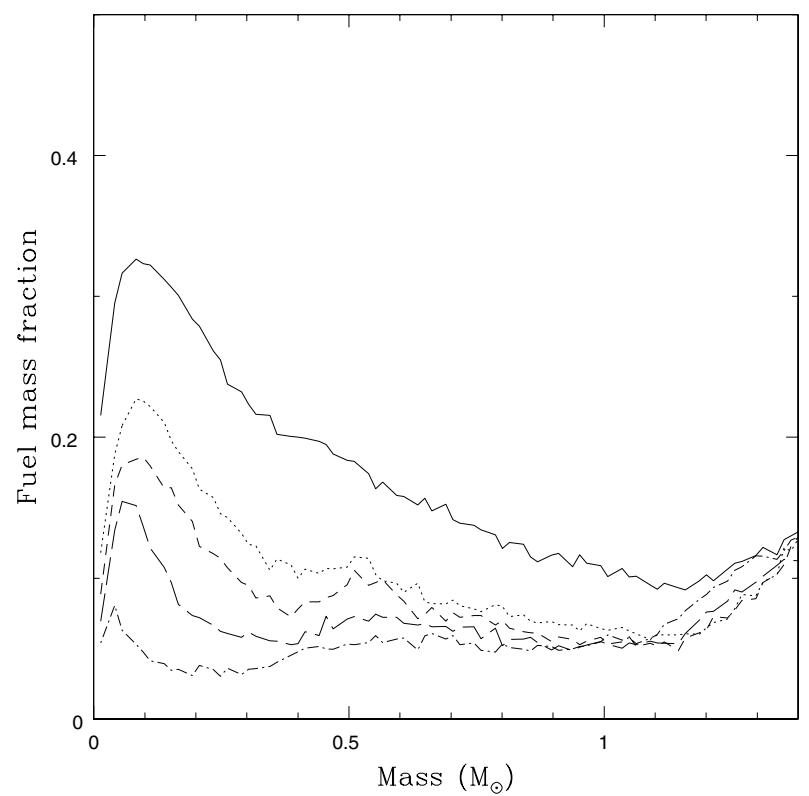

Fig. 8. Evolution of angle-averaged profiles of the ${ }^{12} \mathrm{C}+{ }^{16} \mathrm{O}$ mass fraction during the detonation phase of model DDT3DA. The profile at DDT is shown together with those belonging to the same times shown in Fig. 7.

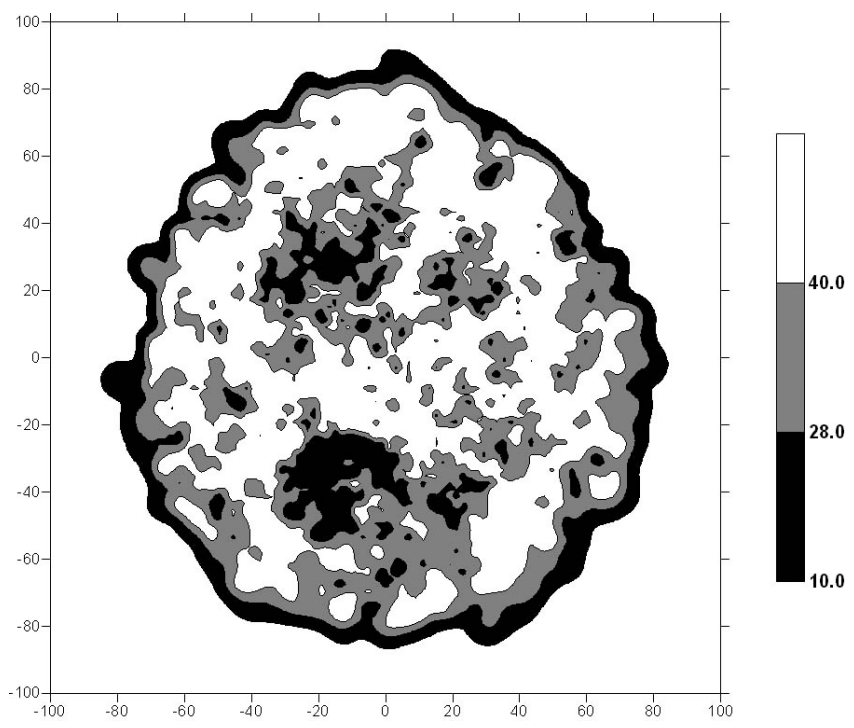

Fig. 9. Map of the final chemical composition on a cut across the ejected matter: $\mathrm{C}-\mathrm{O}$ is shown in black, intermediate-mass elements in grey, and $\mathrm{Fe}-\mathrm{Ni}$ in white. The incinerated matter pervades the whole ejecta, with intermediate-mass elements dominating an annular region at intermediate radius. Unburned $\mathrm{C}-\mathrm{O}$ are found in isolated pockets, mainly in the outer regions of the ejecta. The origin of the large $\mathrm{C}-\mathrm{O}$ pockets seen in this figure can be traced back to features of the flame front identifiable in Figs. 2, 6, and 7.

radioactive ${ }^{56} \mathrm{Ni}$ (Fig. 9). The distribution of species in velocity space (Fig. 10) shows that the ejecta is not chemically stratified, as demanded by recents observations of the light curve in the near and middle infrared (Höflich et al. 2002) as well as by the properties of the X-ray emission of putative type Ia supernova remnants (Badenes et al. 2006). The Fe that makes up most of the ejecta (Fig. 10, see also Fig. 9) is mostly produced during the explosion in the form of radioactive ${ }^{56} \mathrm{Ni}$, whereas the curves labelled as "Ni" trace the distribution of stable iron-group species. Such stable nuclei are nearly absent from the low velocity
Table 3. Nucleosynthesis products, in solar masses.

\begin{tabular}{lcc|lcc}
\hline \hline & DDT3DA & DDT3DB & & DDT3DA & DDT3DB \\
\hline${ }^{12} \mathrm{C}$ & 0.14 & 0.19 & ${ }^{55} \mathrm{Mn}$ & $1.2 \mathrm{E}-3$ & $1.9 \mathrm{E}-3$ \\
${ }^{16} \mathrm{O}$ & 0.25 & 0.26 & ${ }^{54} \mathrm{Fe}$ & 0.061 & 0.055 \\
${ }^{20} \mathrm{Ne}$ & 0.026 & 0.015 & ${ }^{56} \mathrm{Fe}$ & 0.66 & 0.68 \\
${ }^{22} \mathrm{Ne}$ & 0.005 & 0.007 & ${ }^{57} \mathrm{Fe}$ & 0.013 & $6.1 \mathrm{E}-3$ \\
${ }^{24} \mathrm{Mg}$ & 0.014 & 0.008 & ${ }^{59} \mathrm{Co}$ & $3.1 \mathrm{E}-4$ & $1.4 \mathrm{E}-4$ \\
${ }^{28} \mathrm{Si}$ & 0.064 & 0.047 & ${ }^{58} \mathrm{Ni}$ & 0.059 & 0.055 \\
${ }^{32} \mathrm{~S}$ & 0.027 & 0.023 & ${ }^{60} \mathrm{Ni}$ & 0.034 & 0.015 \\
${ }^{36} \mathrm{Ar}$ & $6.1 \mathrm{E}-3$ & $5.6 \mathrm{E}-3$ & ${ }^{61} \mathrm{Ni}$ & $5.6 \mathrm{E}-4$ & $2.2 \mathrm{E}-4$ \\
${ }^{40} \mathrm{Ca}$ & $7.4 \mathrm{E}-3$ & $6.7 \mathrm{E}-3$ & ${ }^{62} \mathrm{Ni}$ & $1.5 \mathrm{E}-3$ & $5.6 \mathrm{E}-4$ \\
${ }^{44} \mathrm{Ca}$ & $9.9 \mathrm{E}-5$ & $2.3 \mathrm{E}-5$ & ${ }^{65} \mathrm{Cu}$ & $3.2 \mathrm{E}-6$ & $1.4 \mathrm{E}-6$ \\
${ }^{48} \mathrm{Ti}$ & $3.1 \mathrm{E}-4$ & $1.9 \mathrm{E}-4$ & ${ }^{64} \mathrm{Zn}$ & $5.0 \mathrm{E}-4$ & $1.9 \mathrm{E}-4$ \\
${ }^{52} \mathrm{Cr}$ & $3.4 \mathrm{E}-3$ & $2.7 \mathrm{E}-3$ & ${ }^{6} \mathrm{Zn}$ & $3.8 \mathrm{E}-5$ & $1.4 \mathrm{E}-5$ \\
\hline
\end{tabular}

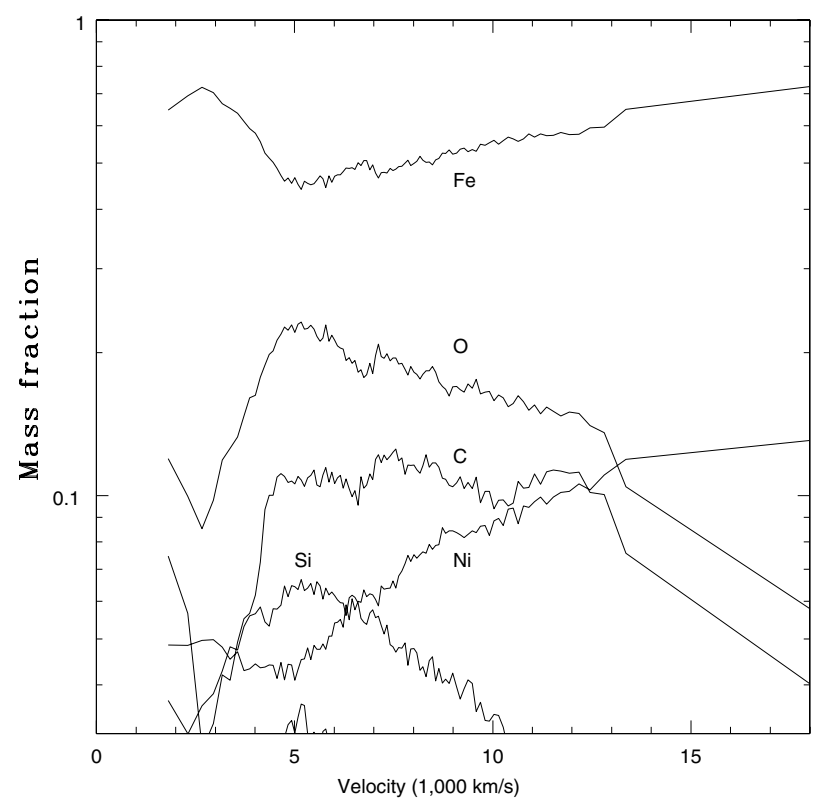

Fig. 10. Final distribution of elements in model DDT3DA as a function of velocity, after radioactive decay.

layers of the ejecta, which does not match the requirements from infrared observations of SNIa (Motohara et al. 2006; Gerardy et al. 2007; Fesen et al. 2007).

\subsection{Sensitivity studies}

We have explored the sensitivity of our results to the parameters of the initial model and to the deflagration-to-detonation transition criterion.

To start with, we changed the wavenumber of the perturbation imposed to the velocity field at the flame location at the beginning of the three-dimensional calculation. Decreasing the wavenumber from $l=20^{3}$ to $l=7$ has virtually no effect on the results of the simulation, as the global figures change by less than $2-3 \%$. Next, we checked the sensitivity to the amplitude of the perturbation. Although this parameter is more influential than the wavenumber, its imprint is still quite modest. Reducing the amplitude from 200 to $100 \mathrm{~km} \mathrm{~s}^{-1}$ translates into a less energetic explosion ( 0.16 foes less than in DDT3DA) and produces

${ }^{3}$ Note that, at $t=0$, the wavelength of the perturbation was $\sim 5$ times the resolution of the code for our reference model with $l=20$. 

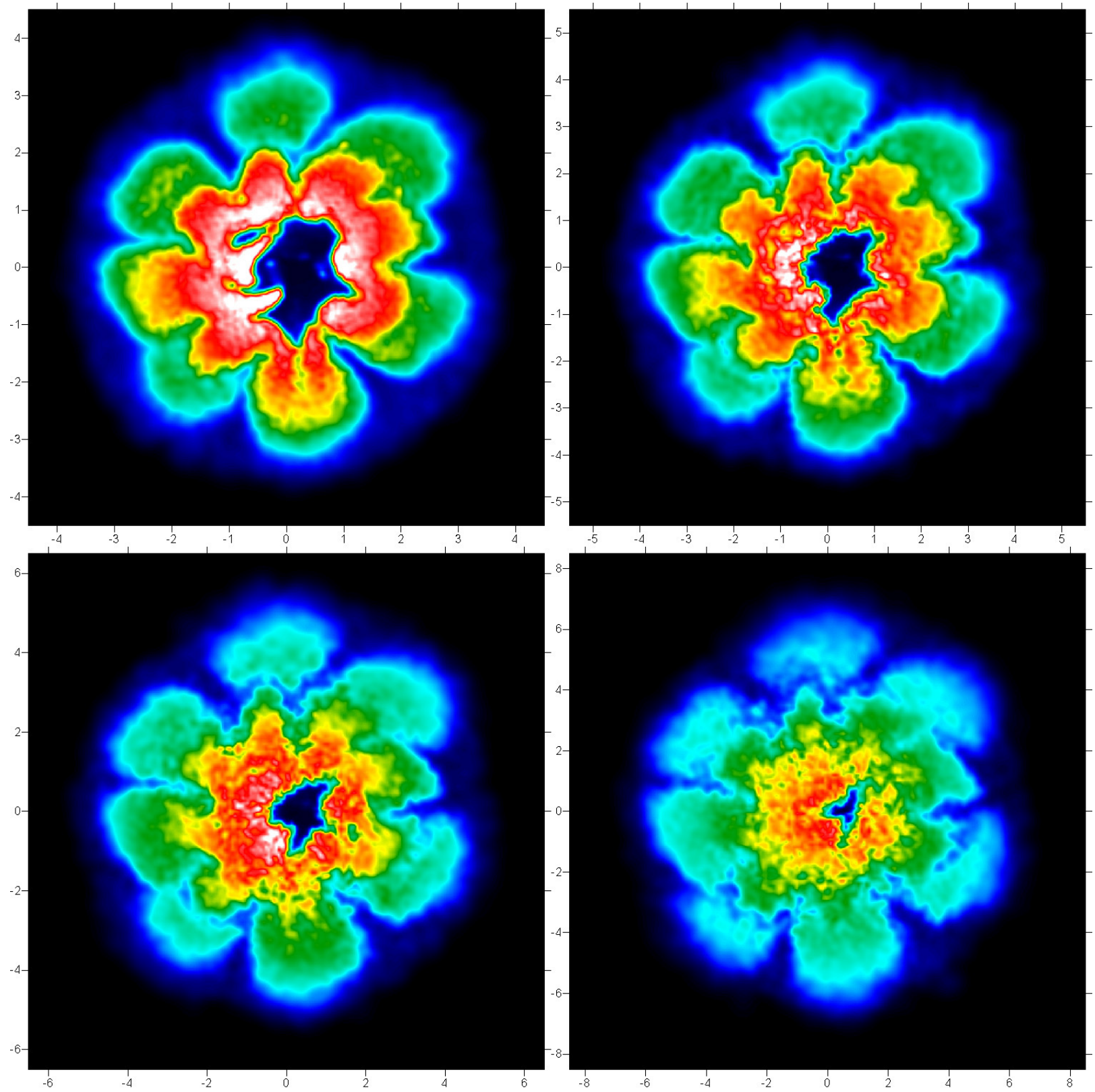

Fig. 11. Snapshots of the temperature map in a cut across the star during the detonation phase of model DDT3DB, at times $t=0.8,0.9,1.0$, and $1.1 \mathrm{~s}$. The scales of length and temperature are the same as in Fig. 2.

$0.11 M_{\odot}$ less of ${ }^{56} \mathrm{Ni}$. Of course, this implies that the remaining mass of unburnt carbon and oxygen is larger than in DDT3DA.

To explore the sensitivity of the results to the DDT criterion we took the same configuration at the end of the deflagration phase as in DDT3DA, but used alternative prescriptions to select the location of the induced detonation:

- Core detonation. All flame particles whose radius was lower than $1.3 \times 10^{8} \mathrm{~cm}$ were instantaneously incinerated. The density in these regions was higher than $\sim 4 \times 10^{7} \mathrm{~g} \mathrm{~cm}^{-3}$.

- Mid-altitude detonation. In this variant all flame particles within radii $1.7 \times 10^{8} \mathrm{~cm}$ and $2.0 \times 10^{8} \mathrm{~cm}$ were instantaneously incinerated. Their mean density was approximately $\sim 2 \times 10^{7} \mathrm{~g} \mathrm{~cm}^{-3}$.

- Atmospheric detonation. All flame particles within $2.4 \times$ $10^{8} \mathrm{~cm}$ and $2.8 \times 10^{8} \mathrm{~cm}$ were instantaneously incinerated. Their densities ranged from $\sim 7 \times 10^{6} \mathrm{~g} \mathrm{~cm}^{-3}$ to $\sim 10^{7} \mathrm{~g} \mathrm{~cm}^{-3}$.
The diversity of supernova explosions obtained with these prescriptions is not as rich as that usually achieved in onedimensional delayed detonations. The kinetic energy varies at most in 0.15 foes, whereas the range of ${ }^{56} \mathrm{Ni}$ masses spans from 0.34 to $0.43 M_{\odot}$. By contrast, one-dimensional models with transition densities in the same range as above produce explosions with kinetic energies that change by 0.34 foes and ${ }^{56} \mathrm{Ni}$ masses ranging from 0.38 to $0.97 M_{\odot}$ (Badenes et al. 2003, 2005). Other properties of these three-dimensional explosions are similar to what was found in DDT3DA: the remaining mass of unburned carbon and oxygen is in all cases too large, and they lack the desired level of chemical stratification.

\subsection{Model DDT3DB}

The evolution of model DDT3DB during the first $0.81 \mathrm{~s}$ belonging to the deflagration phase was identical to that of model B30U 
in García-Senz \& Bravo (2005). At that time a DDT was artificially induced in the vicinity of the flame. The properties of the model at DDT are given in Table 2. In comparison with the configuration of DDT3DA at the moment of transition to detonation, the structure of DDT3DB was more centrally condensed, the central density being a factor $\sim 4$ higher and the average flame density nearly $3.7 \times 10^{7} \mathrm{~g} \mathrm{~cm}^{-3}$. The total energy and incinerated mass are, however, quite similar in both models, as is the amount of unburned fuel. The major difference was in the mass of ${ }^{56} \mathrm{Ni}$ synthesized during the deflagration phase, due to the longest time spent by DDT3DA at high densities, which enabled incinerated matter to experience more electron captures. Consequently, the deflagration phase of model DDT3DB was able to synthesize more ${ }^{56} \mathrm{Ni}$, but less neutronized elements. This difference was still visible in the final results, after the detonation phase, although somewhat attenuated (Table 1).

The temporal evolution of the detonation phase in model DDT3DB is shown in Fig. 11. As can be seen in Fig. 5 of García-Senz \& Bravo (2005, central figure in the right column) at the time of DDT the hot nuclear ashes formed a broken ring that enclosed a central volume rich in carbon and oxygen. The ensuing detonation preferentially propagated inwards processing most of the inner fuel into Fe-peak nuclei without having to deal with major obstacles composed of almost inert matter. However, a detonation wave was not successfully launched outwards and, as a result, the already vigorous expansion of the white dwarf prevented the outer layers from being processed by the nuclear bomb.

Model DDT3DB shares the same problems as DDT3DA: low final kinetic energy, large mass of unburned carbon and oxygen, and lack of chemical stratification (Fig. 12). A significative difference between both delayed detonation models is the amount of neutronized Fe-peak nuclei ejected in the explosion. The mass of slightly neutronized nuclei such as ${ }^{54} \mathrm{Fe}$ and ${ }^{58} \mathrm{Ni}$ was of the same order in both models because their production is determined by the initial presence of ${ }^{22} \mathrm{Ne}(1 \%$ in mass) mixed with ${ }^{12} \mathrm{C}$ and ${ }^{16} \mathrm{O}$, rather than by electron captures on NSE matter. The final ratio of the masses of ${ }^{54} \mathrm{Fe}$ and ${ }^{58} \mathrm{Ni}$ with respect to ${ }^{56} \mathrm{Fe}$ is about 1.5 and 2 times solar respectively. However, the production of more neutronized Fe-peak nuclei in model DDT3DB is more scarce than in DDT3DA due to the rapid migration of the bubbles to low density regions shortly after the beginning of the deflagration phase. Had our initial models started from even higher central densities this effect would had been accentuated.

\section{Conclusions}

The multidimensional delayed detonation model still remains an alternative way to account for the observational properties of type Ia supernovae, although there still remain many pieces of the puzzle to be settled. Among them is the mechanism of transition from deflagration to detonation, especially its viability in an exploding white dwarf. Although the most satisfactory approach to the problem would be to reveal the mechanism from first physical principles, this is an exceedingly difficult task in view of the many different length and temporal scales involved and of the huge diversity of initial conditions that can be imagined. Even though some advances have been brought about (Khokhlov 1991; Khokhlov et al. 1997; Niemeyer \& Woosley 1997; Niemeyer 1999; Lisewski et al. 2000; Röpke et al. 2004; Zingale \& Dursi 2007) no sound mechanism for the transition has yet been found.

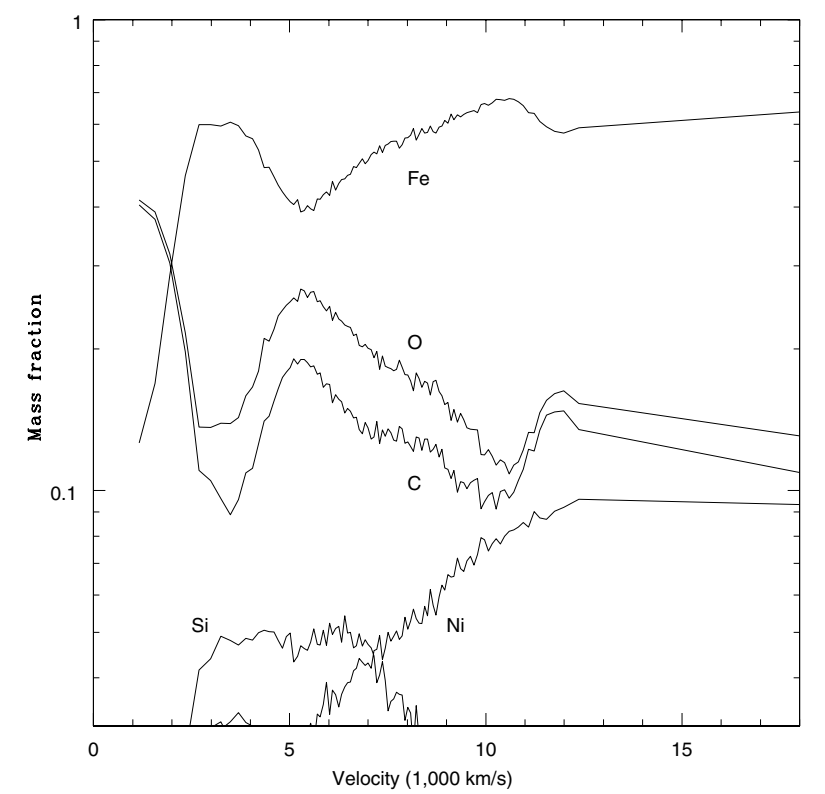

Fig. 12. Final distribution of elements in model DDT3DB as a function of velocity, after radioactive decay.

Given the lack of a consistent physical picture of the DDT mechanism the opposite path is usually taken, trying to constrain the conditions of DDT by comparing the resulting explosion with the observational data of SNIa. However, this approach is encumbered by the vast set of DDT conditions that need to be tested if a three-dimensional model has to be built. In this paper, we have tried to contribute to the still scarce set of three-dimensional simulations of delayed detonation models with two simulations in which the transition takes place at densities in the same range as those favored by succesful onedimensional delayed detonation models of SNIa, i.e. $\rho_{\mathrm{tr}} \sim 1-4 \times$ $10^{7} \mathrm{~g} \mathrm{~cm}^{-3}$.

Our delayed detonation models improve on the pure deflagration ones, but there still remain severe deficiencies compared to observations of SNIa, the two most important being the lack of chemical stratification and the huge mass of unburned carbon and oxygen ejected in the supernova explosion. While the amount of ejected carbon and oxygen might be affected by resolution issues, it is unclear if it can be lowered as much as needed to fit the observations. On the other hand, the lack of chemical stratification may not depend as much on the details of the numerical implementation. To our knowledge, a chemically stratified three-dimensional delayed detonation has only been found in a single work so far (Gamezo et al. 2005), but they had to assume a singularly high density of DDT. The presence of highvelocity nickel above the silicon layer could also be at odds with the observations of SNIa, but this cannot be ascertained in the absence of a three-dimensional spectral calculation.

Another interesting result is the relative insensitivity of the global properties of the explosion with respect to variations in the initial model (geometry of the runaway volume: centered vs bubbly) and location of the DDT (at a fixed transition time). Differences in kinetic energy of 0.15 foes and ejected masses of ${ }^{56} \mathrm{Ni}$ of $0.09 M_{\odot}$ as we obtained are in clear contrast to the rich diversity of explosions obtained with one-dimensional and twodimensional (Arnett \& Livne 1994) delayed detonation models in which the DDT spanned similar density ranges.

Model DDT3DB can be compared with the simulations performed by Röpke \& Niemeyer (2007). In this last work three 
delayed detonation models were presented in which the initial runaway took place in different numbers of hot bubbles, whereas the densities of transition to detonation ranged from 1.3 to $2.4 \times 10^{7} \mathrm{~g} \mathrm{~cm}^{-3}$, compared with the transition density of model DDT3DB, $\rho_{\mathrm{tr}}=3.7 \times 10^{7} \mathrm{~g} \mathrm{~cm}^{-3}$ (Table 2). In spite of having used diverse numerical techniques and slightly different physics in both calculations, the final kinetic energy of our model DDT3DB (0.81 foes) fits quite well in the trend of the asymptotic kinetic energies given by Röpke \& Niemeyer (1.5 to 1.0 foes). The same can be said about the ejected mass of unburned carbon and oxygen, which goes from 0.04 to $0.22 M_{\odot}$ in their models, to be compared with $0.45 M_{\odot}$ of $\mathrm{C}-\mathrm{O}$ ejected in DDT3DB. The convergence of results of these different simulations supports the models reported here.

Acknowledgements. We thank the referee for a careful reading of the manuscript and many positive suggestions that helped to improved the clarity of the text. This work has been partially supported by the MEC grants AYA2005-08013C03, AYA2004-06290-C02, by the European Union FEDER funds and by the Generalitat de Catalunya.

\section{References}

Arnett, D., \& Livne, E. 1994, ApJ, 427, 330

Badenes, C., Bravo, E., Borkowski, K. J., \& Domínguez, I. 2003, ApJ, 593, 358 Badenes, C., Borkowski, K., Bravo, E., Hughes, J. P., \& Hwang, U. 2005, Mem. Soc. Astron. It., 76, 555

Badenes, C., Borkowski, K. J., Hughes, J. P., Hwang, U., \& Bravo, E. 2006, ApJ, 645,1373

Domínguez, I., \& Höflich, P. 2000, ApJ, 528, 854

Fesen, R., Höflich, P., Hamilton, A., et al. 2007, ApJ, 658, 396

Gamezo, V. N., Khokhlov, A. M., Oran, E. S., Chtchelkanova, A. Y., \& Rosenberg, R. O. 2003, Science, 299, 77

Gamezo, V. N., Khokhlov, A. M., \& Oran, E. S. 2004, Phys. Rev. Lett., 92, 211102

Gamezo, V. N., Khokhlov, A. M., \& Oran, E. S. 2005, ApJ, 623, 337

García-Senz, D., \& Bravo, E. 2003, Nucl. Phys. A, 718, 563
García-Senz, D., \& Bravo, E. 2005, A\&A, 430, 585

Garcia-Senz, D., \& Woosley, S. E. 1995, ApJ, 454, 895

Garcia-Senz, D., Bravo, E., \& Serichol, N. 1998, ApJS, 115, 119

García-Senz, D., Bravo, E., \& Woosley, S. E. 1999, A\&A, 349, 177

García-Senz, D., Bravo, E., Cabezón, R. M., \& Woosley, S. E. 2007, ApJ, 660, 509

Gerardy, C. L., Meikle, W. P. S., Kotak, R., et al. 2007, ApJ, 661, 995

Golombek, I., \& Niemeyer, J. C. 2005, A\&A, 438, 611

Hillebrandt, W., \& Niemeyer, J. C. 2000, ARA\&A, 38, 191

Höflich, P., Gerardy, C. L., Fesen, R. A., \& Sakai, S. 2002, ApJ, 568, 791

Ivanova, L. N., Imshennik, V. S., \& Chechetkin, V. M. 1974, Ap\&SS, 31, 497

Khokhlov, A. M. 1988, Ap\&SS, 149, 91

Khokhlov, A. M. 1991, A\&A, 245, 114

Khokhlov, A. M., Oran, E. S., \& Wheeler, J. C. 1997, ApJ, 478, 678

Kozma, C., Fransson, C., Hillebrandt, W., et al. 2005, A\&A, 437, 983

Kuhlen, M., Woosley, S. E., \& Glatzmaier, G. A. 2006, ApJ, 640, 407

Lisewski, A. M., Hillebrandt, W., \& Woosley, S. E. 2000, ApJ, 538, 831

Livne, E. 1999, ApJ, 527, L97

Maier, A., \& Niemeyer, J. C. 2006, A\&A, 451, 207

Motohara, K., Maeda, K., Gerardy, C. L., et al. 2006, ApJ, 652, L101

Niemeyer, J. C. 1999, ApJ, 523, L57

Niemeyer, J. C., \& Kerstein, A. R. 2000, Combust. Sci. Tech., 128, 343

Niemeyer, J. C., \& Woosley, S. E. 1997, ApJ, 475, 740

Reinecke, M., Hillebrandt, W., \& Niemeyer, J. C. 2002, A\&A, 386, 936

Röpke, F. K., \& Niemeyer, J. C. 2007, A\&A, 464, 683

Röpke, F. K., Hillebrandt, W., \& Niemeyer, J. C. 2004, A\&A, 421, 783

Röpke, F. K., Gieseler, M., Reinecke, M., Travaglio, C., \& Hillebrandt, W. 2006a, A\&A, 453, 203

Röpke, F. K., Hillebrandt, W., Niemeyer, J. C., \& Woosley, S. E. 2006b, A\&A, 448, 1

Schmidt, W., \& Niemeyer, J. C. 2006, A\&A, 446, 627

Schmidt, W., Niemeyer, J. C., Hillebrandt, W., \& Röpke, F. K. 2006, A\&A, 450, 283

Timmes, F. X., \& Woosley, S. E. 1992, ApJ, 396, 649

Timmes, F. X., Hoffman, R. D., \& Woosley, S. E. 2000, ApJS, 129, 377

Woosley, S. E., \& Weaver, T. A. 1994, in Supernovae, ed. S. A. Bludman, R. Mochkovitch, \& J. Zinn-Justin, 63

Wunsch, S., \& Woosley, S. E. 2004, ApJ, 616, 1102

Zingale, M., \& Dursi, L. J. 2007, ApJ, 656, 333

Zingale, M., Woosley, S. E., Bell, J. B., Day, M. S., \& Rendleman, C. A. 2005, J. Phys. Conf. Ser., 16, 405 